Article

\title{
A New Flexible Generalized Lindley Model: Properties, Estimation and Applications
}

\author{
Abdulrahman Abouammoh ${ }^{1,+}$ and Mohamed Kayid ${ }^{1,2, *,+}(\mathbb{D}$ \\ 1 Department of Statistics and Operations Research, College of Science, King Saud University, \\ Riyadh 11451, Saudi Arabia; abuammoh@ksu.edu.sa \\ 2 Department of Mathematics and Computer Science, Faculty of Science, Suez University, Suez 43511, Egypt \\ * Correspondence: drkayid@ksu.edu.sa \\ + These authors contributed equally to this work.
}

Received: 28 September 2020; Accepted: 10 October 2020; Published: 14 October 2020

\begin{abstract}
A new method for generalizing the Lindley distribution, by increasing the number of mixed models is presented formally. This generalized model, which is called the generalized Lindley of integer order, encompasses the exponential and the usual Lindley distributions as special cases when the order of the model is fixed to be one and two, respectively. The moments, the variance, the moment generating function, and the failure rate function of the initiated model are extracted. Estimation of the underlying parameters by the moment and the maximum likelihood methods are acquired. The maximum likelihood estimation for the right censored data has also been discussed. In a simulation running for various orders and censoring rates, efficiency of the maximum likelihood estimator has been explored. The introduced model has ultimately been fitted to two real data sets to emphasize its application.
\end{abstract}

Keywords: Lindley distribution; mixture of distributions; gamma distribution; model generalization; right censored data

\section{Introduction}

Lindley [1] distribution has been applied in analyzing lifetime data and stress-strength reliability models, see e.g., Cakmakyapan and Ozel [2]. Many authors have proposed generalizations for the Lindley distribution in recent years. Even so, all of the known generalizations so far have used three main approaches, namely increasing the underlying parameter to two or three, using larger value of the shape parameters and assuming that the parameter of some convenient discrete distributions follows Lindley distribution. The probability density function (PDF) of Lindley is

$$
f(x)=\frac{\theta^{2}}{1+\theta}(1+x) e^{-\theta x}, \quad \theta>0, x \geq 0 .
$$

The well-known Lindley PDF is a mixture of two PDFs $G(1, \theta)$, i.e., gamma with shape parameter 1 and scale $\theta$, and $G(2, \theta)$ with weights $\frac{\theta}{1+\theta}$ and $\frac{1}{1+\theta}$, respectively. The corresponding cumulative distribution function $(\mathrm{CDF})$ is given by

$$
F(x)=1-\frac{1+\theta+\theta x}{1+\theta} e^{-\theta x}, \quad \theta>0, x \geq 0 .
$$

This model was studied in some detail by Ghitany et al. [3] and is a cornerstone in Sankaran [4], Ghitany et al. [5], Zamani and Ismail [6], Ghitany et al. [7], Ghitany et al. [8], Al-Mutairi et al. [9], Al-babtain et al. [10], Ghitany et al. [11] and Al-Mutairi et al. [12]. Moreover, Abouammoh et al. [13] 
have introduced two forms of Lindley generalization by using Lindley with the usual weights $p=\frac{\theta}{1+\theta}$ and $1-p$ and larger value of the underlying shape parameter. Shanker et al. [14] investigated some mathematical properties of an extension of Lindley model.

Many authors improved the flexibility of the Lindley model by increasing the number of parameters. Among them, Shanker and Mishra [15] and Shanker and Ghebretsadik [16] introduced different quasi Lindley models which have one more parameter. Merovci and Sharma [17] introduced the beta-Lindley model which is, in fact, a model with two more parameters. Zakerzade and Dolati [18], Ibrahim et al. [19] and Shanker et al. [20] introduced generalizations of Lindley with three parameters. Also, Broderick and Tiantian [21] proposed a generalized Lindley distribution with four parameters.

Clearly, having proper modelling for data makes improved knowledge of data, gives its characteristics and makes its asymptotic behavior traceable, see for example Wolstenholme [22] and McPherson [23]. However, increasing the number of parameters may provide more flexible models, but it makes the parameter estimation very complicated especially for models with three or more parameters. Therefore, in this paper, a different idea is introduced based on a two-parameter model but applying additional baseline distributions to gain more flexibility. In other words, in this approach, we have one continuous parameter, $\theta$, and one discrete parameter, $m$, and the model is a mixture of $m$ baseline models. $m$ is called the order of mixed distributions.

The sequel of this paper is as follows. In Section 2, the proposed model is introduced, some of its special cases are pointed out and its statistical and reliability properties are investigated. Maximum likelihood estimation of the underlying parameter is discussed in Section 3 and its properties are also studied. The simulation results have been gathered and discussed in Section 4. In the last section, the proposed model has been fitted to some real-life data that have been already studied in the literature for other models.

\section{Definition and Basic Properties}

Here we extend the concept of constructing Lindley PDF as a mixture of two gamma models.

Definition 1. The random variable $X$ is said to have generalized Lindley of order $m$ (GLOm) if its PDF is

$$
f(x)=\frac{\theta^{m}}{\sum_{i=1}^{m} \theta^{m-i}} \sum_{i=1}^{m} \frac{x^{m-i}}{\Gamma(m-i+1)} e^{-\theta x}
$$

This model is in fact a mixture of $m$ gamma distribution (see Cheng and Feast [24]) as

$$
f(x)=\sum_{i=1}^{m} w_{i} g_{i}(x)
$$

where $g_{i}$ is the gamma distribution with parameters $(m-i+1, \theta)$ and the density

$$
g_{i}(x)=\frac{\theta^{m-i+1}}{\Gamma(m-i+1)} x^{m-i} e^{-\theta x}
$$

and $w_{i}=\frac{\theta^{i-1}}{\sum_{i=1}^{m} \theta^{m-i}}$. Please note that for $\theta=1$, all mixed PDFs are given the same weight, namely $\frac{1}{m}$. As $\theta<1$ and decreases more weights are to PDFs with higher shape parameter, whereas $\theta>1$ and increases then more weights are given to PDFs with less shape parameter. Also, for general $m$, the CDF of GLOm is

$$
F(x)=\frac{1}{\sum_{i=1}^{m} \theta^{m-i}} \sum_{i=1}^{m} \theta^{m-i} \frac{\gamma(i, \theta x)}{\Gamma(i)},
$$


where $\gamma(a, x)=\int_{0}^{x} t^{a-1} e^{-t} d t$ is the lower incomplete gamma function. By some algebra, we can write the CDF of GLOm as a simpler expression which since is free from incomplete gamma function is more convenient for numeric computations.

$$
F(x)=1-\frac{\sum_{j=0}^{m-1} \sum_{i=1}^{m-j} \theta^{m-i} \frac{x^{j}}{j !}}{\sum_{i=1}^{m} \theta^{m-i}} e^{-\theta x} .
$$

To check accuracy of (6), we can apply induction on $m$. Clearly, it holds for $m=1$. It is straightforward to show that if we assume (6) is true for $m=k$, then it also holds for $m=k+1$.

Similarly, the survival function can be written as

$$
S(x)=\frac{1}{\sum_{i=1}^{m} \theta^{m-i}} \sum_{i=1}^{m} \frac{\theta^{m-i} \gamma_{u}(i, \theta x)}{\Gamma(i)},
$$

where $\gamma_{u}(a, x)=\int_{x}^{\infty} t^{a-1} e^{-t} d t$ is the upper incomplete gamma function. Also, the $k$ th moment of GLOm can be derived as

$$
\begin{aligned}
E\left(X^{k}\right) & =\int_{0}^{\infty} x^{k} \frac{\theta^{m}}{\sum_{i=1}^{m} \theta^{m-i}} \sum_{i=1}^{m} \frac{x^{m-i}}{\Gamma(m-i+1)} e^{-\theta x} d x \\
& =\left(\sum_{i=1}^{m} \theta^{m-i}\right)^{-1} \sum_{i=1}^{m} \int_{0}^{\infty} \frac{\theta^{m}}{\Gamma(m-i+1)} x^{m-i+k} e^{-\theta x} d x \\
& =\frac{\sum_{i=1}^{m}(m-i+k) \ldots(m-i+1) \theta^{i-k-1}}{\sum_{i=1}^{m} \theta^{m-i}} .
\end{aligned}
$$

When $k$ vanishes to 1 or 2 , it reduces to

$$
E(X)=\frac{\sum_{i=1}^{m}(m-i+1) \theta^{i-2}}{\sum_{i=1}^{m} \theta^{m-i}}
$$

and

$$
E\left(X^{2}\right)=\frac{\sum_{i=1}^{m}(m-i+2)(m-i+1) \theta^{i-3}}{\sum_{i=1}^{m} \theta^{m-i}},
$$

respectively and can be applied to reach variance of GLOm. The moment generating function (refer to Bulmer [25]) is

$$
\begin{aligned}
m_{X}(t) & =E\left(e^{t X}\right) \\
& =\int_{0}^{\infty} e^{t x} \frac{\theta^{m}}{\sum_{i=1}^{m} \theta^{m-i}} \sum_{i=1}^{m} \frac{x^{m-i}}{\Gamma(m-i+1)} e^{-\theta x} d x \\
& =\left(\sum_{i=1}^{m} \theta^{m-i}\right)^{-1} \sum_{i=1}^{m} \int_{0}^{\infty} \frac{\theta^{m}}{\Gamma(m-i+1)} x^{m-i} e^{-(\theta-t) x} d x \\
& =\frac{\theta^{m}}{\sum_{i=1}^{m} \theta^{m-i}} \sum_{i=1}^{m}(\theta-t)^{-m+i-1} \\
& =\frac{\sum_{i=1}^{m}(\theta-t)^{-i}}{\sum_{i=1}^{m} \theta^{-i}} .
\end{aligned}
$$

The failure rate function of this model is

$$
h(x)=\frac{f(x)}{S(x)}=\frac{\theta^{m} \sum_{i=1}^{m} \frac{x^{m-i}}{\Gamma(m-i+1)}}{\sum_{j=0}^{m-1} \sum_{i=1}^{m-j} \theta^{m-i} \frac{x^{j}}{j !}} .
$$




\section{Comparison of GLOm for Small Ms}

To elucidate some properties of GLOm it would be interesting to consider basic statistical properties for small values of $m$ such as $m=2,3$ and 4. Although it is known that for $m=1$ the GLOm is reduced to exponential, Table 1 shows the PDF, CDF, mean and failure rate function for small $m s$. For a detailed description of failure rate, we can refer to Lai and Xie [26].

Table 1. PDF, CDF, mean and failure rate for generalized Lindley random variables of orders 2,3 and 4.

\begin{tabular}{cccc}
\hline & GLO2 & GLO3 & GLO4 \\
\hline PDF & $\frac{\theta^{2}}{1+\theta}(1+x) e^{-\theta x}$ & $\frac{\theta^{3}}{1+\theta+\theta^{2}}\left(1+x+\frac{1}{2} x^{2}\right) e^{-\theta x}$ & $\frac{\theta^{4}}{1+\theta+\theta^{2}+\theta^{3}}\left(1+x+\frac{1}{2} x^{2}+\frac{1}{6} x^{3}\right) e^{-\theta x}$ \\
\hline CDF & $1-\left(\frac{1+\theta+\theta x}{1+\theta}\right) e^{-\theta x}$ & $1-\left(\frac{\left(1+\theta+\theta^{2}\right)+\left(\theta+\theta^{2}\right) x+\left(\theta^{2}\right) \frac{x^{2}}{2}}{1+\theta+\theta^{2}}\right) e^{-\theta x}$ & $1-\left(\frac{\left(1+\theta+\theta^{2}+\theta^{3}\right)+\left(\theta+\theta^{2}+\theta^{3}\right) x+\left(\theta^{2}+\theta^{3}\right) \frac{x^{2}}{2}+\left(\theta^{3}\right) \frac{x^{3}}{6}}{1+\theta+\theta^{2}+\theta^{3}}\right)$ \\
\hline Mean & $\frac{2+\theta}{\theta+\theta^{2}}$ & $\frac{3+2 \theta+\theta^{2}}{\theta+\theta^{2}+\theta^{3}}$ & $\frac{4+3 \theta+2 \theta^{2}+\theta^{3}}{\theta+\theta^{2}+\theta^{3}+\theta^{4}}$ \\
\hline \multirow{2}{*}{ Failure rate } & $\frac{\theta^{2}(1+x)}{1+\theta+\theta x}$ & $\frac{\theta^{3}\left(1+x+\frac{x^{2}}{2}\right)}{\left(1+\theta+\theta^{2}\right)+\left(\theta+\theta^{2}\right) x+\left(\theta^{2}\right) \frac{x^{2}}{2}}$ & $\frac{\theta^{4}\left(1+x+\frac{x^{2}}{2}+\frac{x^{3}}{6}\right)}{\left(1+\theta+\theta^{2}+\theta^{3}\right)+\left(\theta+\theta^{2}+\theta^{3}\right) x+\left(\theta^{2}+\theta^{3}\right) \frac{x^{2}}{2}+\left(\theta^{3}\right) \frac{x^{3}}{6}}$ \\
\hline
\end{tabular}

Figures 1-3 draw density, survival and failure rate functions for some $\theta$ and $m$ values. One can note that as $\theta$ decreases the mode drift away from zero in GLOm, for $m=2,3,4$ and 5. Furthermore, the PDF shape is more skewed to the left as the order $m$ decreases.

Please note that the survival, in Figure 2 decreases sharper as the order $m$ decreases and as $\theta$ increases. Consequently, Figure 3 shows that the failure rate increases sharper for smaller $m$ and larger $\theta$.
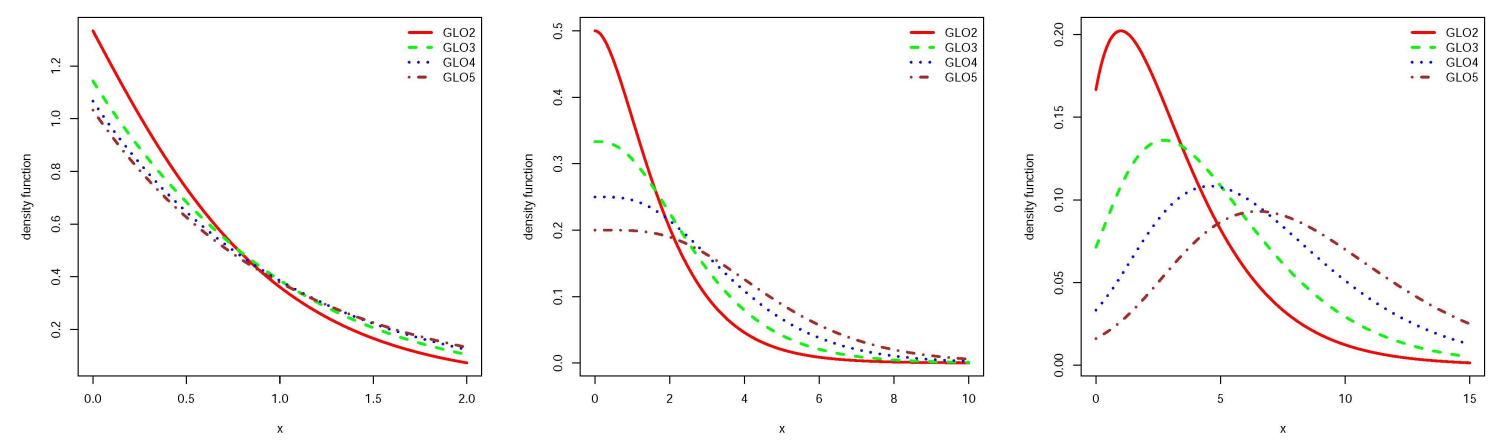

Figure 1. Density function for GLO2, GLO3, GLO4 and GLO5 and $\theta=2,1$ and 0.5 from left to right respectively.
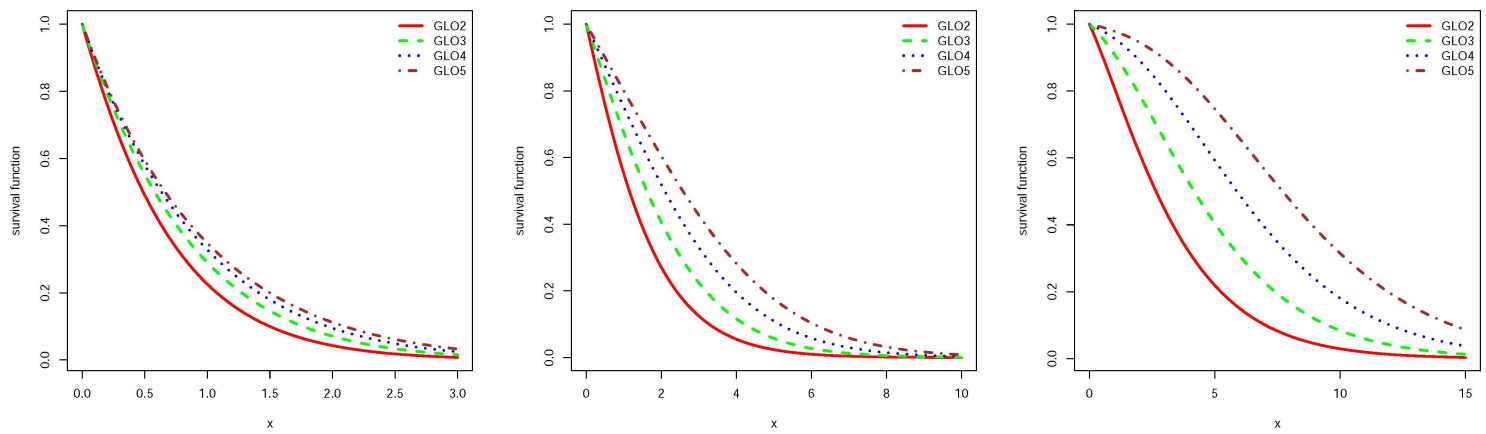

Figure 2. Survival function for GLO2, GLO3, GLO4 and GLO5 and $\theta=2,1$ and 0.5 from left to right respectively. 

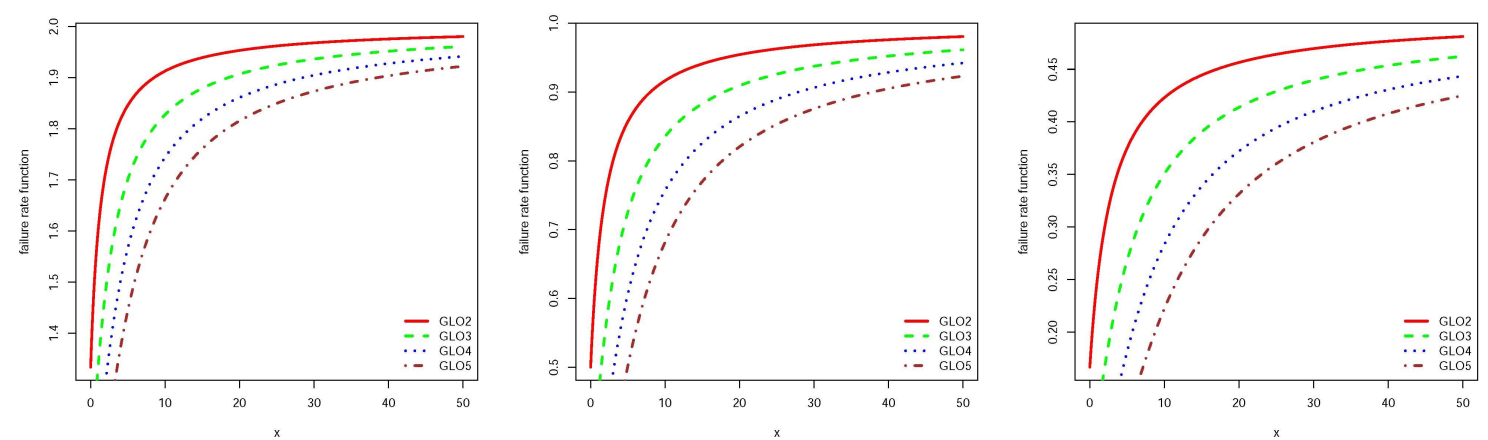

Figure 3. Failure rate function for GLO2, GLO3, GLO4 and GLO5 and $\theta=2,1$ and 0.5 from left to right respectively.

\section{Estimation of the Parameters}

Consider one sample of size $n$ following GLOm denoted by $X_{1}, X_{2}, \ldots, X_{n}$. For known $m$, to provide an estimation of $\theta$ via the moments method, we can apply the equation

$$
\left(\sum_{i=1}^{m} \theta^{m-i}\right)^{-1} \sum_{i=1}^{m}(m-i+1) \theta^{i-2}=\bar{X} .
$$

It is an equation of degree $m$ for $\theta$ which gives $m$ potential answers for $\theta$. Then, we may have up to $m$ different estimations for $\theta$. We can use these estimation values of $\theta$ as initial values for optimizing likelihood function.

For outcomes $x_{1}, x_{2}, \ldots, x_{n}$ of GLOm distribution, when $m$ is known, the log-likelihood function is

$$
l(\theta)=n m \ln \theta-n \ln \left(\sum_{i=1}^{m} \theta^{m-i}\right)-\theta \sum_{j=1}^{n} x_{j}+\sum_{j=1}^{n} \ln \left(\sum_{i=1}^{m} \frac{x_{j}^{m-i}}{\Gamma(m-i+1)}\right) .
$$

The last expression does not depend on $\theta$ and can be ignored in the optimization process. However, it must be taken into account for comparing different models in terms of their likelihood values. When $m$ is unknown, which is usually the case, we optimize the likelihood function for $m=1,2,3, \ldots$ and compare the likelihood values to find a proper $m$.

By differentiation from (10), the score statistics for $m \geq 2$, is given as

$$
\frac{d l(\theta)}{d \theta}=\frac{n m}{\theta}-n \frac{\sum_{i=1}^{m-1}(m-i) \theta^{m-i-1}}{\sum_{i=1}^{m} \theta^{m-i}}-\sum_{j=1}^{n} X_{j}
$$

Therefore, the Fisher information for $\theta$ and $m=2$ is

$$
E\left(-\frac{d^{2} l(\theta)}{d \theta^{2}}\right)=\frac{2 n}{\theta^{2}}-\frac{n}{(1+\theta)^{2}}
$$

and for $m \geq 3$

$$
\begin{aligned}
E\left(-\frac{d^{2} l(\theta)}{d \theta^{2}}\right) & =\frac{n m}{\theta^{2}} \\
& -n \frac{\left(\sum_{i=1}^{m-2}(m-i)(m-i-1) \theta^{m-i-2}\right)\left(\sum_{i=1}^{m} \theta^{m-i}\right)}{\left(\sum_{i=1}^{m} \theta^{m-i}\right)^{2}} \\
& +n \frac{\left(\sum_{i=1}^{m-1}(m-i) \theta^{m-i-1}\right)^{2}}{\left(\sum_{i=1}^{m} \theta^{m-i}\right)^{2}} .
\end{aligned}
$$


It is well-known that variance of the maximum likelihood estimator is reverse of the Fisher information. For more details of the maximum likelihood estimation, see Shao [27].

\section{Right Censored Data}

Let $X_{i}, i=1,2, \ldots, n$ follow the GLOm model representing independent event times which are exposed to right random censoring. The $i$ th event time $X_{i}$ is said to be censored, when it occurs after corresponding random censorship time $C_{i}$, i.e., whenever $C_{i} \leq X_{i}$. In this situation, the censoring time is assumed to be observed and the only information about the event time is that it is greater than the observed censoring time. Thus, the observations are supposed to be consisting of $T_{i}=\min \left(X_{i}, C_{i}\right)$ and $\Delta_{i}=I\left[X_{i} \leq C_{i}\right]$, where $\Delta_{i}$ is said to be censoring indicator.

For more information about the right censoring phenomenon in survival data, we refer the readers to Fleming and Harrington [28].

In presence of data in the specific form as $\left(t_{i}, \delta_{i}\right)$ where $t_{i}=\min \left(x_{i}, c_{i}\right)$, the log-likelihood function is obtained as

$$
l(\theta)=\sum_{i=1}^{n} \delta_{i} \ln f\left(t_{i}\right)+\sum_{i=1}^{n}\left(1-\delta_{i}\right) \ln S\left(t_{i}\right),
$$

in which $f$ and $S$ are, respectively, density and survival functions of the GLOm.

\section{Simulation}

In accordance with the certitude that the GLOm is veritably a mixture of gamma distributions, the following couple of steps can be taken to generate a sample of size $n$ from the GLOm.

1. Simulate one random variable of multinomial distribution with parameters $n$ and $w_{i}, i=$ $1,2, \ldots, m, \sum_{i=1}^{m} w_{i}=1$. Assume the generated instance be denoted by $k_{1}, k_{2}, \ldots, k_{m}$ corresponding respectively to probabilities $w_{1}, w_{2}, \ldots, w_{m}$. Please note that $\sum_{i=1}^{m} k_{i}=n$.

2. Simulate samples of sizes $k_{i}, i=1,2, \ldots, m$ from gamma distribution with parameters $(m-i+$ $1, \theta)$. Then, we can merge these samples to provide one sample of size $n$ from GLOm model.

The results of our simulation study have been gathered in Table 2. Each time, proper values of $m$ and $\theta$ is selected and $r=500$ replicates of samples of size $n=20$ have been drawn. For each replicate, the maximum likelihood estimation $(\hat{\theta})$ has been computed. Then, three measures

$$
\begin{gathered}
B=\frac{1}{r} \sum_{i=1}^{r}(\hat{\theta}-\theta), \\
B^{*}=\frac{1}{r} \sum_{i=1}^{r}|\hat{\theta}-\theta|,
\end{gathered}
$$

and

$$
M S E=\frac{1}{r} \sum_{i=1}^{r}(\hat{\theta}-\theta)^{2},
$$

have been reported in the table. The simulation results reported in Table 2 indicate two points.

- As $m$ increases, $B, B^{*}$ and $M S E$ of $\hat{\theta}$ decrease.

- Also, $B, B^{*}$ and MSE increase with $\theta$. 
Table 2. Simulation results for estimating $\hat{\theta}$.

\begin{tabular}{ccccc}
\hline $\boldsymbol{m}$ & $\boldsymbol{\theta}$ & $\boldsymbol{B}$ & $\boldsymbol{B}^{*}$ & $\boldsymbol{M S E}$ \\
\hline 2 & 0.01 & 0.000392 & 0.001285 & 0.000002 \\
& 0.2 & 0.003687 & 0.027083 & 0.001223 \\
& 2 & 0.089519 & 0.303592 & 0.156634 \\
\hline 3 & 0.01 & 0.000212 & 0.001064 & 0.000001 \\
& 0.2 & 0.003756 & 0.021395 & 0.000725 \\
& 2 & 0.065359 & 0.260616 & 0.113436 \\
\hline 4 & 0.01 & 0.000223 & 0.000956 & 0.000001 \\
& 0.2 & 0.002531 & 0.018226 & 0.000538 \\
& 2 & 0.067082 & 0.234948 & 0.090523 \\
\hline 5 & 0.01 & 0.000036 & 0.000827 & 0.000001 \\
& 0.2 & 0.001806 & 0.016431 & 0.000420 \\
& 2 & 0.048145 & 0.205068 & 0.072493 \\
\hline 6 & 0.01 & 0.000089 & 0.000762 & 0.000000 \\
& 0.2 & 0.000261 & 0.014639 & 0.000346 \\
& 2 & 0.048055 & 0.203754 & 0.068692 \\
\hline
\end{tabular}

\section{Censored Data}

In another simulation study, $p=0.25$ and $p=0.40$ of the sample simulated from the GLOm have been censored from right. In actuality, the random censorship time $C$ was assumed to follow the uniform distribution on $[0, u]$. Given the value of $p$, the amount of $u$ is the root of the equation

$$
\int_{0}^{u} S(t) d t=u p,
$$

in which $S(t)$ is the survival function of the GLOm. We solve this equation for both values of $p$ mentioned above and generate $r=500$ replicates of samples of sizes $n=20,40$. For each replicate, the maximum likelihood method is applied to perceive the estimation of $\theta$. The results of the simulation study have been gathered in Table 3 for $p=0.25$ and in Table 4 for $p=0.40$.

As a summary of the simulation, we observe and verify the following points:

- $\quad$ The mean of the absolute bias $\left(B^{*}\right)$ is slightly greater than that for the complete (non-censored) data and increases with rising the portion of the censored part of the sample.

- For small $\theta$ s, in the presence of censorship, the bias exhibits more fluctuation around zero and is more skewed to left. It may, therefore, cause smaller values for $B$ and larger values for $B^{*}$. For large $\theta \mathrm{s}, \hat{\theta}$ is less than the actual value of the parameter and it causes $B$ to be negative and $B^{*}$ being its absolute value.

- Analogously as dealing with non-censored data, when $m$ increases ( $\theta$ decreases), $B, B^{*}$ and MSE of $\hat{\theta}$ decrease. 
Table 3. Simulation results for estimating $\hat{\theta}$ when $25 \%$ of the sample has been censored from right.

\begin{tabular}{|c|c|c|c|c|c|}
\hline$n$ & $m$ & $\theta$ & $B$ & $B^{*}$ & $M S E$ \\
\hline \multirow[t]{12}{*}{20} & 2 & 0.01 & 0.000228 & 0.001437 & 0.000003 \\
\hline & & 0.2 & 0.005887 & 0.029532 & 0.001384 \\
\hline & & 1.2 & -0.218137 & 0.218137 & 0.055005 \\
\hline & 3 & 0.01 & 0.000192 & 0.001251 & 0.000002 \\
\hline & & 0.2 & 0.001745 & 0.022546 & 0.000809 \\
\hline & & 1.2 & -0.209398 & 0.209398 & 0.044895 \\
\hline & 4 & 0.01 & 0.000066 & 0.000936 & 0.000001 \\
\hline & & 0.2 & 0.002496 & 0.019698 & 0.000614 \\
\hline & & 1.2 & -0.204693 & 0.204693 & 0.042309 \\
\hline & 5 & 0.01 & 0.000041 & 0.000888 & 0.000001 \\
\hline & & 0.2 & 0.001541 & 0.018230 & 0.000541 \\
\hline & & 1.2 & -0.202124 & 0.202124 & 0.040991 \\
\hline \multirow[t]{12}{*}{40} & 2 & 0.01 & 0.000041 & 0.001018 & 0.000001 \\
\hline & & 0.2 & 0.002479 & 0.020986 & 0.000699 \\
\hline & & 1.2 & -0.206282 & 0.206282 & 0.043137 \\
\hline & 3 & 0.01 & 0.000012 & 0.000769 & 0.000001 \\
\hline & & 0.2 & 0.001470 & 0.015627 & 0.000389 \\
\hline & & 1.2 & -0.202902 & 0.202902 & 0.041378 \\
\hline & 4 & 0.01 & 0.000029 & 0.000694 & 0.000000 \\
\hline & & 0.2 & 0.001004 & 0.014321 & 0.000313 \\
\hline & & 1.2 & -0.201060 & 0.201060 & 0.040472 \\
\hline & 5 & 0.01 & 0.000002 & 0.000636 & 0.000000 \\
\hline & & 0.2 & -0.000065 & 0.012032 & 0.000226 \\
\hline & & 1.2 & -0.200265 & 0.200265 & 0.040113 \\
\hline
\end{tabular}

Table 4. Simulation results for estimating $\hat{\theta}$ when $40 \%$ of the sample has been censored from right.

\begin{tabular}{cccccc}
\hline$n$ & $\boldsymbol{m}$ & $\boldsymbol{\theta}$ & $\boldsymbol{B}$ & $\boldsymbol{B}^{*}$ & $\boldsymbol{M S E}$ \\
\hline 20 & 2 & 0.01 & 0.000307 & 0.001546 & 0.000003 \\
& & 0.2 & 0.003416 & 0.032657 & 0.001677 \\
& & 1.2 & -0.221936 & 0.221936 & 0.053017 \\
\hline & 3 & 0.01 & 0.000057 & 0.001298 & 0.000002 \\
& 0.2 & 0.000150 & 0.024646 & 0.000981 \\
& 1.2 & -0.216420 & 0.216420 & 0.049195 \\
\hline \multirow{2}{*}{4} & 0.01 & -0.000017 & 0.001050 & 0.000002 \\
& 0.2 & 0.001599 & 0.021568 & 0.000731 \\
& 1.2 & -0.206033 & 0.206033 & 0.043004 \\
\hline 5 & 0.01 & -0.000027 & 0.000901 & 0.000001 \\
& 0.2 & 0.001679 & 0.018990 & 0.000585 \\
& 1.2 & -0.203726 & 0.203726 & 0.041973 \\
\hline 40 & 2 & 0.01 & 0.000093 & 0.001088 & 0.000002 \\
& 0.2 & 0.000000 & 0.021989 & 0.000781 \\
& 1.2 & -0.211623 & 0.211623 & 0.046143 \\
\hline 3 & 0.01 & 0.000072 & 0.000867 & 0.000001 \\
& 0.2 & -0.000216 & 0.016417 & 0.000432 \\
& 1.2 & -0.205086 & 0.205086 & 0.042572 \\
\hline 4 & 0.01 & -0.000024 & 0.000739 & 0.000000 \\
& 0.2 & 0.000730 & 0.014284 & 0.000318 \\
& 1.2 & -0.202220 & 0.202220 & 0.041028 \\
\hline 5 & 0.01 & 0.000014 & 0.000652 & 0.000000 \\
& 0.2 & -0.000495 & 0.012862 & 0.000261 \\
& 1.2 & -0.201258 & 0.201258 & 0.040596 \\
\hline
\end{tabular}




\section{Real Data Examples}

\subsection{Failure of Yarn}

Here, we consider one real data set reported by Lawless [29]. The data set which shows the number of cycles to failure for 25 specimens yarn have been gathered in Table 5 .

Table 5. Number of cycles to failure for 25 specimens yarn.

\begin{tabular}{ccccccccccccc}
\hline 15 & 20 & 38 & 42 & 61 & 76 & 86 & 98 & 121 & 146 & 149 & 157 & 175 \\
176 & 180 & 180 & 198 & 220 & 224 & 251 & 264 & 282 & 321 & 325 & 653 & \\
\hline
\end{tabular}

The results of fitting GLOm distribution to this data set is presented in Table 6 for $m=1$ to 4 . Clearly, the likelihood is stronger for $m=2$. Therefore, we prefer GLO2, among others. Figure 4 shows empirical survival function along fitted models.

Table 6. Estimate of $\theta$ and -log-likelihood for GLOm with different $m$ values.

\begin{tabular}{cccc}
\hline $\boldsymbol{m}$ & $\hat{\boldsymbol{\theta}}$ & $\boldsymbol{v a \hat { r }}(\hat{\boldsymbol{\theta}})$ & -log-likelihood \\
\hline 1 & 0.00560 & 0.00000125 & 154.5895 \\
2 & 0.01116 & 0.00000249 & 152.5070 \\
3 & 0.01674 & 0.00000373 & 154.5319 \\
4 & 0.02230 & 0.00000497 & 158.1863 \\
\hline
\end{tabular}

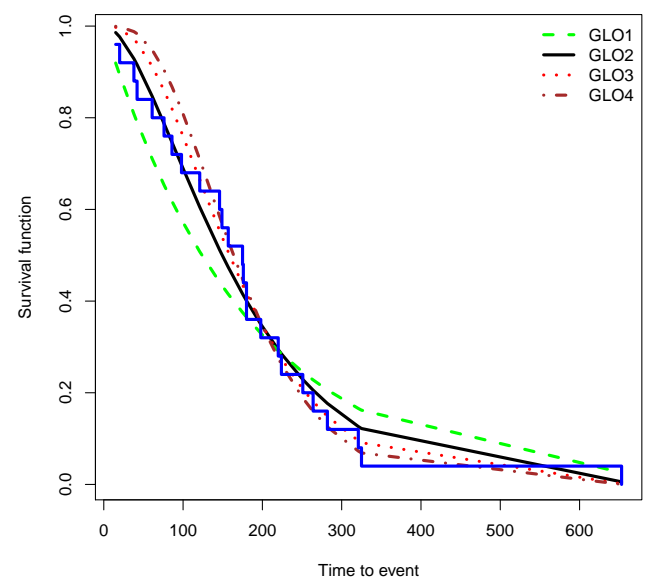

Figure 4. Empirical survival function and survival function of fitted GLOm.

\subsection{Ovarian Cancer}

Edmunson et al. [30] considered survival data related to some ovarian cancer patients. The data set has also been reported in 'survival' package in $\mathrm{R}$ and includes variables 'futime' indicating time to death or censoring of the patients and 'fustat' as censoring status. Table 7 shows results of fitting data to GLOm for $m=1,2,3$ and 4. Based on the log-likelihood measure, among these models, GLO3 describe the data better. The Kaplan-Meier survival function along with GLOms have been drawn in Figure 5.

Table 7. Estimate of $\theta$ and -log-likelihood for GLOm with different $m$ values.

\begin{tabular}{cccc}
\hline $\boldsymbol{m}$ & $\hat{\boldsymbol{\theta}}$ & $\boldsymbol{v} \hat{\boldsymbol{a}} \boldsymbol{r}(\hat{\boldsymbol{\theta}})$ & -log-likelihood \\
\hline 1 & 0.00118 & 0.00000019 & 92.8365 \\
2 & 0.00316 & 0.00000028 & 90.0980 \\
3 & 0.00529 & 0.00000057 & 89.7396 \\
4 & 0.00752 & 0.00000085 & 90.2579 \\
\hline
\end{tabular}




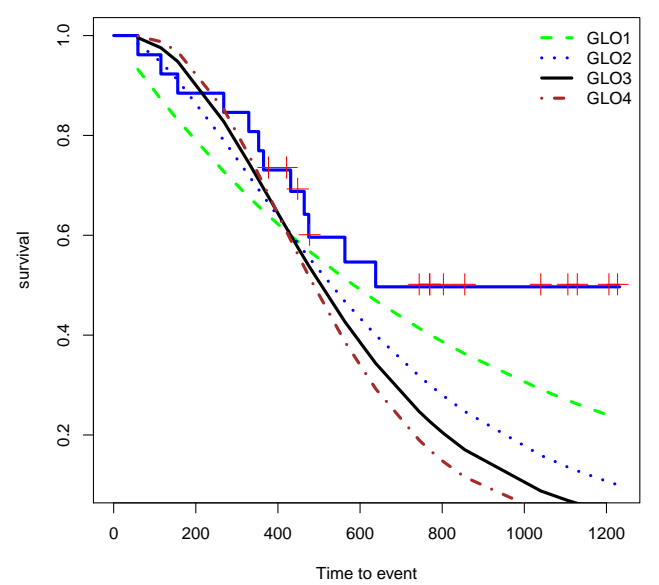

Figure 5. Kaplan-Meier survival function and survival function of fitted GLOm.

\section{Conclusions}

Lindley distribution which is a mixture of gamma models has attracted the attention of many researchers in recent decades. It has been shown that this distribution is quite useful in describing real data sets. Over and above that, extensions of this model have also been introduced in the literature. Here, we introduced a fresh method to generalize the Lindley distribution which the flexibility rises by increasing the number of mixed components in the model. The proposed model is called the GLOm where $m$ represents the number of baseline models called the order of the model. We studied some properties of GLOm for different $m$. The moments, the variance and the moment generating function were shown to have closed forms for every $m$. The MLE of the parameter have been discussed for either of the right censored data and the complete data. The supplied simulation results indicated that the MLE is suitable for both the censored data and the non-censored data. Two real data sets were discussed showing that generalizing the Lindley distribution as accomplished in this paper may be helpful to describe the data more conveniently.

Author Contributions: Formal analysis, A.A. and M.K.; Investigation, M.K.; Methodology, A.A. and M.K.; Software, A.A.; Writing-original draft, A.A. All authors have read and agreed to the published version of the manuscript.

Funding: This Project was funded by the National Plan for Science, Technology and Innovation (MAARIFAH), King Abdulaziz City for Science and Technology, Kingdom of Saudi Arabia, Award Number (14-MAT2052-02).

Acknowledgments: The authors are grateful to anonymous referees for their constructive comments that lead to an improvement in the quality of the paper. The authors would like to extend their sincere appreciation to the strategic technology program of the National Plan for Science, Technology and Innovation in the Kingdom of Saudi Arabia for its funding this project No. 14-MAT2052-02.

Conflicts of Interest: There is not conflict of interest.

\section{References}

1. Lindley, D.V. Fiducial distributions and Bayes' theorem. J. R. Stat. Soc. Ser. (Methodol.) 1958, 20, $102-107$. [CrossRef]

2. Cakmakyapan, S.; Ozel, G. The Lindley family of distributions: Properties and applications. Hacet. J. Math. Stat. 2017, 46, 1113-1137. [CrossRef]

3. Ghitany, M.E.; Atieh, B.; Nadarajah, S. Lindley distribution and its application. Math. Comput. Simul. 2008, 78, 493-506. [CrossRef]

4. Sankaran, M. The discrete poisson-Lindley distribution. Biometrics 1970, 26, 145-149. [CrossRef]

5. Ghitany, M.E.; Al-Mutairi, D.K.; Nadarajah, S. Zero-truncated Poisson-Lindley distribution and its application. Math. Comput. Simul. 2008, 79, 279-287. [CrossRef]

6. Zamani, H.; Ismail, N. Negative Binomial-Lindley Distribution and Its Application. J. Math. Stat. 2010, 6, 4-9. [CrossRef] 
7. Ghitany, M.E.; Al-Mutairi, D.K.; Awadhi, F.A.; Alburais, M. Marshall-Olkin extended Lindley distribution and its application. Int. J. Appl. Math. 2012, 25, 709-721.

8. Ghitany, M.E.; Al-Mutairi, D.K.; Balakrishnan, N.; Al-Enezi, L.J. Power Lindley distribution and associated inference. Comput. Stat. Data Anal. 2013, 64, 20-33. [CrossRef]

9. Al-Mutairi, D.K.; Ghitany, M.E.; Kundu, D. Inferences on stress-strength reliability from Lindley distribution. Commun. Stat.-Theory Methods 2013, 42, 1443-1463. [CrossRef]

10. Al-babtain, A.A.; Eid, H.A.; A-Hadi, N.A.; Merovci, F. The five parameter Lindley distribution. Pak. J. Stat. 2014, 31, 363-384.

11. Ghitany, M.E.; Al-Mutairi, D.K.; Aboukhamseen, S.M. Estimation of the reliability of a stress-strength system from power Lindley distributions. Commun. Stat.-Simul. Comput. 2015, 44, 118-136. [CrossRef]

12. Al-Mutairi, D.K.; Ghitany, M.E.; Kundu, D. Inferences on stress-strength reliability from weighted lindley distributions. Commun. Stat.-Theory Methods 2015, 44, 4096-4113. [CrossRef]

13. Abouammoh, A.M.; Alshangiti Arwa, M.; Ragab, I.E. A new generalized Lindley distribution. J. Stat. Comput. Simul. 2015, 85, 3662-3678. [CrossRef]

14. Shanker, R.; Fesshaye, H.; Sharma, S. On Two-Parameter Lindley Distribution and its Applications to Model Lifetime Data. Biom. Biostat. Int. J. 2016, 1, 9-15. [CrossRef]

15. Shanker, R.; Mishra, A. A quasi Lindley distribution. Afr. J. Math. Comput. Sci. Res. 2013, 6, 64-71.

16. Shanker, R.; Ghebretsadik, A.H. A New Quasi Lindley Distribution. Int. J. Stat. Syst. 2013, 8, $143-156$.

17. Merovci, F.; Sharma, V.K. The Beta-Lindley Distribution: Properties and Applications. J. Appl. Math. 2014, 2014, 198951. [CrossRef]

18. Zakerzadeh, H.; Dolati, A. Generalized Lindley distribution. J. Math. Ext. 2009, 3, 13-25.

19. Ibrahim, E.; Merovci, F.; Elgarhy, M. A new generalized Lindley distribution. Math. Theory Model. 2013, 3, 30-47.

20. Shanker, R.; Shukla, K.K.; Shanker, R.; Leonida, T.A. A Three-Parameter Lindley Distribution. Am. J. Math. Stat. 2017, 7, 15-26.

21. Broderick, O.O.; Tiantian, Y. A new class of generalized Lindley distributions with applications. J. Stat. Comput. Simul. 2015, 85, 2072-2100. [CrossRef]

22. Wolstenholme, L.C. Reliability Modelling: A Statistical Approach; Chapman and Hall/CRC: London, UK, 1999; ISBN 9781584880141.

23. McPherson, J.W. Reliability Physics and Engineering: Time-To-Failure Modeling; Springer: Berlin/Heidelberg, Germany, 2010; ISBN 978-1-4419-6348-2.

24. Cheng, R.; Feast, G. Some Simple Gamma Variate Generators. J. R. Stat. Soc. Ser. C (Appl. Stat.) 1979, 28, 290-295. [CrossRef]

25. Bulmer, M.G. Principles of Statistics; Dover: New York, NY, USA, 1979; ISBN 0-486-63760-3.

26. Lai, C.D.; Xie, M. Stochastic Aging and Dependence for Reliability; Springer: New York, NY, USA, 2006; ISBN 978-0-387-29742-2.

27. Shao, J. Mathematical Statistics; Springer: New York, NY, USA, 2003. [CrossRef]

28. Fleming, T.R.; Harrington, D.P. Counting Processes and Survival Analysis; Wiley: Hoboken, NJ, USA, 2011; ISBN 978-1-118-15066-5.

29. Lawless, J.F. Statistical Models and Methods for Lifetime Data, 2nd ed.; Wiley: Hoboken, NJ, USA, 2003.

30. Edmunson, J.H.; Fleming, T.R.; Decker, D.G.; Malkasian, G.D.; Jefferies, J.A.; Webb, M.J.; Kvols, L.K. Different Chemotherapeutic Sensitivities and Host Factors Affecting Prognosis in Advanced Ovarian Carcinoma vs. Minimal Residual Disease. Cancer Treat. Rep. 1979, 63, 241-247.

Publisher's Note: MDPI stays neutral with regard to jurisdictional claims in published maps and institutional affiliations.

(C) 2020 by the authors. Licensee MDPI, Basel, Switzerland. This article is an open access article distributed under the terms and conditions of the Creative Commons Attribution (CC BY) license (http://creativecommons.org/licenses/by/4.0/). 